\title{
Complement C5a induces mesenchymal stem cell apoptosis during the progression of chronic diabetic complications
}

\author{
Ming Zhu ${ }^{1} \cdot$ Xiao $\mathrm{He}^{1} \cdot$ Xiao-Hui Wang $^{1,2} \cdot$ Wei Qiu $^{1} \cdot$ Wei Xing $^{1} \cdot$ Wei Guo ${ }^{1}$. \\ Tian-Chen $\mathrm{An}^{1} \cdot$ Luo-Quan Ao ${ }^{1} \cdot$ Xue-Ting $\mathrm{Hu}^{1} \cdot \mathrm{Zhan}^{1} \mathbf{~}^{1} \cdot \mathrm{Xiao}^{1}$-Ping Liu ${ }^{2}$ • \\ Nan $\mathrm{Xiao}^{3} \cdot$ Jian $\mathrm{Yu}^{4,5} \cdot$ Hong Huang ${ }^{1} \cdot$ Xiang $\mathrm{Xu}^{1}$
}

Received: 8 November 2016 / Accepted: 25 April 2017 / Published online: 3 June 2017

(C) Springer-Verlag Berlin Heidelberg 2017

\begin{abstract}
Aims/hypothesis Regeneration and repair mediated by mesenchymal stem cells (MSCs) are key self-protection mechanisms against diabetic complications, a reflection of diabetes-related cell/tissue damage and dysfunction. MSC abnormalities have been reported during the progression of diabetic complications, but little is known about whether a deficiency in these cells plays a role in the pathogenesis of this disease. In addition to MSC resident sites, peripheral circulation is a major source of MSCs that participate in the regeneration and repair of damaged tissue. Therefore, we investigated whether there is
\end{abstract}

Electronic supplementary material The online version of this article (doi:10.1007/s00125-017-4316-1) contains peer-reviewed but unedited supplementary material, which is available to authorised users.

Xiang Xu

xiangxu@ymail.com

Hong Huang

huanghongcq@163.com

1 First Department, State Key Laboratory of Trauma, Burn and Combined Injury, Daping Hospital and Research Institute of Surgery, Third Military Medical University, No. 10 Changjiang Branch Road, Daping Street, Yuzhong District, Chongqing 400042, People's Republic of China

2 Department of Histology and Embryology, Medical College of Qingdao University, Qingdao, People's Republic of China

3 Ninth Department, Daping Hospital and Research Institute of Surgery, Third Military Medical University, Chongqing, People's Republic of China

4 Department of Pathology, University of Pittsburgh School of Medicine, Pittsburgh, PA, USA

5 University of Pittsburgh Cancer Institute, University of Pittsburgh School of Medicine, Pittsburgh, PA, USA a deficiency of circulating MSC-like cells in people with diabetes and explored the underlying mechanisms.

Methods The abundance of MSC-like cells in peripheral blood was evaluated by FACS. Selected diabetic and nondiabetic serum (DS and NDS, respectively) samples were used to mimic diabetic and non-diabetic microenvironments, respectively. The proliferation and survival of MSCs under different serum conditions were analysed using several detection methods. The survival of MSCs in diabetic microenvironments was also investigated in vivo using leptin receptor mu$\operatorname{tant}\left(\right.$ Lepr $\left.^{d b / d b}\right)$ mice.

Results Our data showed a significant decrease in the abundance of circulating MSC-like cells, which was correlated with complications in individuals with type 2 diabetes. DS strongly impaired the proliferation and survival of cultureexpanded MSCs through the complement system but not through exposure to high glucose levels. DS-induced MSC apoptosis was mediated, at least in part, by the complement C5a-dependent upregulation of Fas-associated protein with death domain (FADD) and the Bcl-2-associated X protein $(\mathrm{BAX}) / \mathrm{B}$ cell lymphoma 2 (Bcl-2) ratio, which was significantly inhibited by neutralising $\mathrm{C} 5 \mathrm{a}$ or by the pharmacological or genetic inhibition of the C5a receptor (C5aR) on MSCs. Moreover, blockade of the $\mathrm{C} 5 \mathrm{a} / \mathrm{C} 5 \mathrm{aR}$ pathway significantly inhibited the apoptosis of transplanted MSCs in eepr $^{d b / d b}$ recipient mice.

Conclusions/interpretation C5a-dependent apoptotic death is probably involved in MSC deficiency and in the progression of complications in individuals with type 2 diabetes. Therefore, anticomplement therapy may be a novel intervention for diabetic complications.

Keywords Apoptosis $\cdot \mathrm{C} 5 \mathrm{a} / \mathrm{C} 5 \mathrm{aR}$ pathway $\cdot$ Complement $\cdot$ Complication · Deficiency $\cdot$ Mesenchymal stem cell · Type 2 diabetes 


$\begin{array}{ll}\text { Abbreviations } \\ \text { BAX } & \text { Bcl-2-associated X protein } \\ \text { Bcl-2 } & \text { B cell lymphoma 2 } \\ \text { C5aR } & \text { C5a receptor } \\ \text { CH50 } & \text { 50\% complement haemolytic activity } \\ \text { CI } & \text { Cell index } \\ \text { DS } & \text { Diabetic serum } \\ \text { FADD } & \text { Fas-associated protein with death domain } \\ \text { FPG } & \text { Fasting plasma glucose } \\ \text { HI-DS } & \text { Heat-inactivated diabetic serum } \\ \text { HI-NDS } & \text { Heat-inactivated non-diabetic serum } \\ \text { hUC-MSC } & \text { Human umbilical cord-derived } \\ & \text { mesenchymal stem cell } \\ \text { MAC } & \text { Membrane attack complex } \\ \text { mBM-MSC } & \text { Mouse bone marrow-derived } \\ & \text { mesenchymal stem cell } \\ \text { MSC } & \text { Mesenchymal stem cell } \\ \text { MTT } & \text { 3-(4,5-Dimethylthiazol-2-yl)-2,5- } \\ & \text { diphenyltetrazolium bromide } \\ \text { NDS } & \text { Non-diabetic serum } \\ \text { PBMC } & \text { Peripheral blood mononuclear cell } \\ \text { PB-MSC } & \text { Peripheral blood-derived mesenchymal } \\ & \text { stem cell } \\ \text { RTCA } & \text { Real-time cell analysis } \\ \text { sC5b-9 } & \text { Soluble C5b-9 complex } \\ \text { WT } & \text { Wild-type }\end{array}$

\section{Introduction}

The global prevalence of diabetes has nearly doubled since 1980 , increasing from $4.7 \%$ to $8.5 \%$ in the adult population, and an estimated 422 million adults were living with diabetes in 2014 [1,2]. The number of people with diabetes is estimated to increase to 552 million worldwide by 2030 [3], and one in ten adults will have diabetes by 2040 [4]. As a major cause of death and disability worldwide, diabetes places a heavy burden on individuals, families and the healthcare system [1, 4-6].

People with diabetes have a high risk of developing a series of related complications, including diabetic cardiomyopathy, retinopathy, neuropathy, foot ulcers and nephropathy [7]. The risk factors and mechanisms involved in the pathogenesis of complications in individuals with diabetes are complex and have not been totally defined $[8,9]$; thus, the prevention, treatment and reversal of diabetic complications are restricted.

Diabetic complications are a reflection of metabolic disorder-induced cell/tissue damage and dysfunction, with manifestations in multiple organs. In view of the essential roles of mesenchymal stem cells (MSCs) in target tissue regeneration and repair [10-13], we may reasonably speculate that there must be quantitative, distributive or functional anomalies in endogenous MSCs in individuals with diabetes who present complications that impair the ability of these cells to regenerate and repair the damaged tissue. Indeed, a pathogenic role for impaired MSC quality in diabetes has been proposed by many researchers [14-18], and a decreased number of MSCs has also been reported to be associated with this disease $[19,20]$; however, the causes and role of MSC deficiency in diabetes are unknown.

When tissue is damaged, resident MSCs, which rapidly function as seed cells, are recruited to the injury site and a large number of MSCs are also recruited from the peripheral circulation to participate in the regeneration and repair of the injured tissue $[18,19]$. Therefore, we speculate that the number of circulating MSCs in individuals with diabetes might be decreased, ultimately resulting in an insufficient number of these cells at the injury site and the impaired regeneration and repair of target tissues. Moreover, both hyperglycaemia and excessive complement activation are characteristics of diabetes that have been reported to be involved in the pathogenesis of diabetic complications [9, 21-24]; thus, we investigated their correlations with the abundance of circulating MSCs in this study.

\section{Methods}

\section{Ethics statement}

All work was conducted in full compliance with ethical principles, and prior approval was obtained from the Ethics Committee of Daping Hospital, Third Military Medical University (Chongqing, China). Human samples were collected after obtaining informed consent from the donors or their legal representatives, and the mouse experiments conformed to ethical standards.

\section{Participants and serum collection}

Individuals with type 2 diabetes and age- and sex-matched healthy donors were recruited from Daping Hospital. The demographic and clinical data are provided in Table 1 and Electronic Supplementary Material (ESM) Table 1. Serum samples from all participants were collected and stored according to the principles proposed by Lachmann [25] (see ESM Methods for details).

\section{Genetically modified mice}

Genetic mouse models of diabetes B6.BKS(D)-Lepr ${ }^{\mathrm{db}} / \mathrm{J}$ (Jackson Laboratory, Bar Harbor, ME, USA) and C5a receptor (C5aR)-deficient $\left(\mathrm{Cd} 88^{-/-}\right)$mice $\left(\mathrm{C}^{\mathrm{aral}} \mathrm{I}^{\text {tmlCge}}\right.$; BALB/c background; Jackson Laboratory; please note $C d 88$ is an alias of $C 5 \mathrm{ar} 1$ ) were kindly provided by Y. Wang (Department of Medical Genetics, Third Military Medical University) and G. 
Table 1 Demographic and clinic characteristics of the diabetic and control participants

\begin{tabular}{|c|c|c|c|c|c|}
\hline \multirow[t]{2}{*}{ Characteristic } & \multirow[t]{2}{*}{ Control group $(n=15)$} & \multicolumn{3}{|c|}{ Type 2 diabetes group $(n=49)$} & \multirow[t]{2}{*}{$p$ value } \\
\hline & & 0 complication $(n=14)$ & 1 complication $(n=19)$ & $\geq 2$ complications $(n=16)$ & \\
\hline Age (years) & $63.5 \pm 5.2$ & $62.2 \pm 5.8$ & $63.4 \pm 4.5$ & $65.6 \pm 4.1$ & 0.290 \\
\hline Sex (male/female) & $9 / 6$ & $8 / 6$ & $11 / 8$ & $9 / 7$ & 0.997 \\
\hline BMI $\left(\mathrm{kg} / \mathrm{m}^{2}\right)$ & $23.3 \pm 1.7$ & $25.9 \pm 1.7 * *$ & $24.6 \pm 1.6^{*}$ & $23.8 \pm 2.3$ & 0.002 \\
\hline Duration of diabetes (years) & NA & $3.3 \pm 1.3$ & $8.7 \pm 1.7^{\dagger}$ & $15.2 \pm 3.6^{\dagger}$ & $<0.001$ \\
\hline $\mathrm{FPG}(\mathrm{mmol} / \mathrm{l})$ & $4.6 \pm 0.6$ & $8.1 \pm 0.6^{* * *}$ & $8.8 \pm 1.2 * * *$ & $9.9 \pm 1.3 * * *$ & $<0.001$ \\
\hline $\mathrm{HbA}_{1 \mathrm{c}}(\%)$ & $4.5 \pm 0.8$ & $7.7 \pm 0.7 * * *$ & $8.5 \pm 0.5^{* * *}$ & $9.1 \pm 0.9 * * *$ & $<0.001$ \\
\hline $\mathrm{HbA}_{1 \mathrm{c}}(\mathrm{mmol} / \mathrm{mol})$ & $25.5 \pm 8.3$ & $60.8 \pm 7.6$ & $69.1 \pm 5.5$ & $75.8 \pm 10.1$ & \\
\hline
\end{tabular}

Data are presented as means \pm SD

All data (excluding sex) were analysed using one-way ANOVA, followed by Dunnett's test for multiple comparisons; sex was analysed using $\chi^{2}$. $* p<0.05, * * p<0.01$ and ${ }^{* * *} p<0.001$ compared with control participants; ${ }^{\dagger} p<0.001$ compared with diabetic individuals with no complications NA, not applicable

$\mathrm{Xu}$ (Institute of Immunology, College of Basic Medical Science, Third Military Medical University), respectively. For details regarding housing, genotyping, breeding methods and the use of control mice, see ESM Methods.

\section{Quantitative detection of MSC-like cells in peripheral blood}

Approximately $4 \mathrm{ml}$ of anticoagulated blood was harvested to analyse the cell subgroups. Peripheral blood mononuclear cells (PBMCs) were isolated by density gradient centrifugation within $2 \mathrm{~h}$ of sample collection. Then, the proportion and number of MSC-like (CD34 $\left.{ }^{-} \mathrm{CD} 105^{+}\right)$cells among PBMCs were determined by FACS analysis. Experimenters were blind to group allocation and outcome assessment. See ESM Methods for details.

For similar detection in mice, anticoagulant peripheral blood was resuspended in commercial ACK lysis buffer (Beyotime, Haimen, China) to lyse the erythrocytes. Then, the nucleated cells were stained and analysed to determine the abundance of MSC-like cells.

\section{Isolation and culture of MSCs}

Human umbilical cord-derived MSCs (hUC-MSCs) were isolated using the tissue block implantation method (see ESM Methods for details). For subcultures of hUC-MSCs, the culture medium was replaced with DMEM/F12 (HyClone, Logan, UT, USA) containing 10\% FBS (Gibco, Grand Island, NY, USA).

Mouse bone marrow-derived MSCs (mBM-MSCs) were isolated using the simple plastic adherent method (see ESM Methods for details).

In this study, hUC-MSCs were used between the second and fifth passages, and mBM-MSCs were used between the fourth and sixth passages. The MSCs were identified by their morphology, surface markers and multi-directional differentiation ability (ESM Fig. 1; see ESM Methods for details).

\section{Cell viability assay}

When the inoculated hUC-MSCs in 96-well plates had attached to the bottom of the plates or were in the midlogarithmic growth phase, the medium was replaced with DMEM/F12 containing different concentrations of D-glucose (Sigma-Aldrich, St Louis, MO, USA) or different types of sera. After treatment for the indicated period, cell viability was measured using the 3-(4,5-dimethylthiazol-2-yl)-2,5-diphenyltetrazolium bromide (MTT) assay, and the relative cell viability was calculated. See ESM Methods for details.

\section{Determination and quantification of apoptotic death}

TUNEL method Single-cell suspensions of hUC-MSCs were seeded on coverslips in a 12-well plate. During the midlogarithmic growth phase, the cells were rinsed and further incubated under different culture conditions. After $12 \mathrm{~h}$, the hUC-MSCs on the coverslips were fixed with $4 \%$ paraformaldehyde (PFA), permeabilised with Triton X-100, stained using a TUNEL Andy Fluor 488 Apoptosis Detection Kit (GeneCopoeia, Guangzhou, China), and further stained with DAPI (Sigma-Aldrich). After being washed with PBS, the cells on coverslips were photographed using a fluorescence microscope (Olympus IX71, Tokyo, Japan).

FACS analysis MSCs in mid-logarithmic growth phase in six-well plates were rinsed and incubated under different culture conditions for $12 \mathrm{~h}$. Then, both the adherent and suspended cells were collected and double stained with annexin V-FITC/PI (KeyGen Biotech, Nanjing, China). Cell 
apoptosis was measured within $30 \mathrm{~min}$ by FACS. Both early (annexin V-FITC ${ }^{+} \mathrm{PI}$ ) and late (annexin $\mathrm{V}^{-} \mathrm{FITC}^{+} \mathrm{PI}^{+}$) apoptotic cells were included in the cell death analyses.

Real-time cell analysis A real-time cell analysis (RTCA) system (ACEA Biosciences, Hangzhou, China) that can dynamically measure impedance-based signals and present the signals as cell index (CI) values was used to continuously monitor the effects of different culture conditions on the adherence and proliferation of hUC-MSCs. See ESM Methods for details.

\section{Complement haemolytic activity and serum levels of complement components}

Human serum samples were thawed rapidly from $-196^{\circ} \mathrm{C}$, and the $50 \%$ complement haemolytic activity $(\mathrm{CH} 50)$ was determined using the red cell haemolysis test (see ESM Methods for details). The levels of complement $\mathrm{C} 3$ and $\mathrm{C} 4$ were measured by technicians at the Department of Clinical Laboratory, Daping Hospital, using the immunoturbidimetry method. The soluble C5b-9 complex (sC5b-9) and C5a were assessed in an enzyme immunoassay (EIA) using the MicroVue C5a EIA and sC5b-9 Plus EIA kits (Quidel; San Diego, CA, USA) according to the manufacturer's instructions.

\section{Western blotting analysis}

MSCs were seeded into $60 \mathrm{~mm}$ dishes and treated with different types of sera when they reached $70 \%$ confluence. After $12 \mathrm{~h}$, the total proteins were extracted, quantified, denatured, separated, immunoblotted and visualised. See ESM Methods and ESM Table 2 for details.

\section{Immunocytochemical analysis}

PE-conjugated anti-human and FITC-conjugated anti-mouse CD88 (C5aR1) antibodies (1:50 dilutions; Cedarlane; Hornby, ON, Canada) were used to identify and locate CD88 in hUCMSCs and mBM-MSCs, respectively. See ESM Methods for details.

\section{Labelling, implantation and detection of mBM-MSCs}

Isolation of mBM-MSCs was performed using wild-type (WT) and $C d 88^{-/}$BALB/c mice (male; 4-6 weeks old). WT and $\mathrm{Lepr}^{d b / d b}$ C57BL/6 mice (male; 8 months old) were used to evaluate the effects of diabetes and the $\mathrm{C} 5 \mathrm{a} / \mathrm{C} 5 \mathrm{aR}$ pathway on the survival of mBM-MSCs in vivo. Briefly, pre-labelled mBM-MSCs were implanted into WT or Lepr ${ }^{d b / d b}$ mice and their survival was detected by FACS. See ESM Methods for details.

\section{Data analysis}

GraphPad Prism 6 (GraphPad Software, San Diego, CA, USA) was used for data analysis. Data are expressed as means $\pm \mathrm{SD}$ or means $\pm \mathrm{SEM}$ as indicated. Comparisons between groups were analysed using $t$ tests (two-sided; unpaired) or ANOVA. Linear regression and Pearson's correlation analyses were performed to determine whether a relationship existed between two variables. A $p$ value of $<0.05$ was considered to be statistically significant.

\section{Results}

\section{Numbers of circulating MSC-like cells are decreased} in individuals with diabetes and in $L e p r^{d b / d b}$ mice

The average numbers (mean [range]) of $\mathrm{CD} 34^{-} \mathrm{CD} 105^{+}$ MSC-like cells present in $1 \times 10^{5}$ PBMCs were $338(154$ 522 ) in the healthy control participants, 203 (86-321) in diabetic participants without any complications, 141 (47-272) in individuals with one complication, and 123 (28-231) in individuals with multiple complications (Fig. 1a). These results indicated an obviously lower abundance of circulating MSClike cells in the participants with diabetes. Furthermore, we divided the participants with diabetes into five groups according to the number of complications they presented and calculated the average number of circulating MSC-like cells for each group. The results revealed a negative correlation between the number of complications and the abundance of circulating MSC-like cells (Fig. 1b). Those with a longer duration of diabetes or a higher fasting plasma glucose (FPG) levels also tended to have a lower abundance of circulating MSC-like cells (Fig. 1c,d). The lower abundance of these cells was confirmed in Lepr ${ }^{d b / d b}$ mice (Fig. 1e).

\section{DS impairs the proliferation and survival of hUC-MSCs in vitro}

We evaluated the effects of the diabetic microenvironment on the fate of MSCs in vitro to explore the mechanisms underlying the diabetes-related lower abundance of circulating MSClike cells. As predicted, DS (randomly selected serum samples from individuals with multiple diabetic complications) significantly impaired the proliferation and survival of hUC-MSCs compared with NDS (serum samples from age- and sexmatched healthy control participants) (Fig. 2a, b). The cell cycle progression and apoptosis in hUC-MSCs stimulated by the different serum treatments were further assessed, and we found that DS induced cell cycle arrest (ESM Fig. 2) and apoptotic cell death (Fig. 2c-g). 
Fig. 1 Quantitative analysis of the number of circulating MSClike $\left(\mathrm{CD} 34^{-} \mathrm{CD} 105^{+}\right)$cells and correlation with the number of diabetic complications, duration of diabetes and FPG levels. (a) Numbers of circulating MSC-like cells among $1 \times 10^{5}$ PBMCs in diabetic and control participants. (b) Analysis of the correlation between the number of complications and the average number of circulating MSC-like cells in individuals with diabetes. $(\mathbf{c}, \mathbf{d})$ Linear regression analysis of the number of circulating MSC-like cells and duration of diabetes (c) or FPG levels (d). (e) Numbers of circulating MSC-like cells in $L e p r^{d b / d b}$ and control mice ( $n=5$ for each group). $* p<0.05$, $* * * p<0.001$
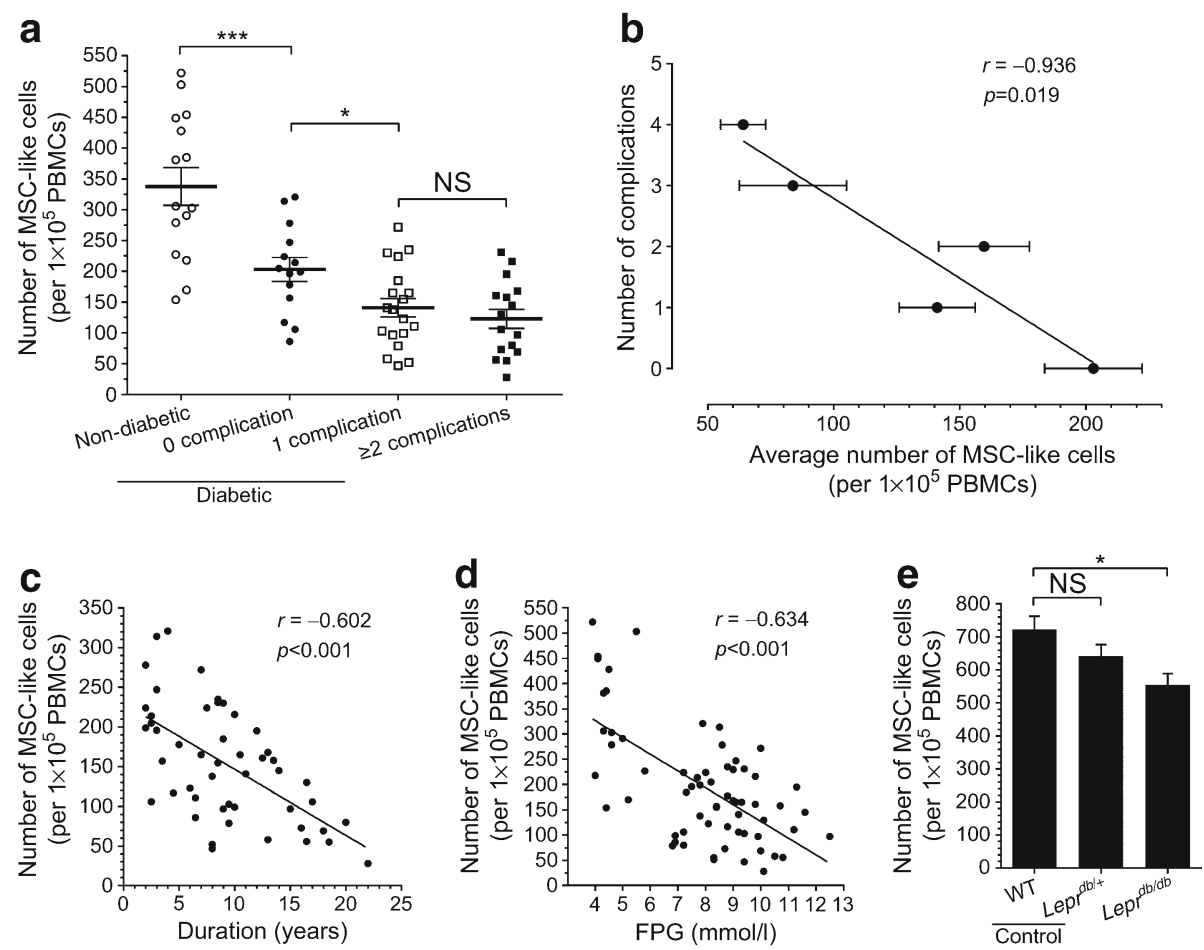

\section{High glucose levels promote hUC-MSC proliferation but have little effect on cell survival}

We assessed the proliferation and survival of hUC-MSCs cultured under different concentrations of D-glucose to investigate whether the diabetes-related MSC deficiency is attributed to high glucose levels. The results showed that high glucose levels promoted the proliferation and cell cycle progression of hUC-MSCs (Fig. 3a, b and ESM Fig. 3) and slightly increased cell apoptosis, but only when the concentration reached $30 \mathrm{mmol} / \mathrm{l}$ (Fig. 3c, d), which is a relatively high blood glucose level that is not typically observed in most diabetic individuals. We adjusted the glucose concentration in NDS to the concentration detected in DS by adding D-glucose and then compared the apoptosis of hUC-MSCs treated with NDS, DS or adjusted NDS to further exclude a role for high glucose levels in the pro-apoptotic effect of DS. The increase in the glucose concentration in NDS did not increase its cytotoxicity. However, DS substantially increased apoptosis compared with adjusted NDS, although these serum samples contained equal glucose concentrations (Fig. 3e, f). Thus, DS must have contained other anomalous factors in addition to high glucose levels that contributed to its cytotoxicity towards hUC-MSCs in vitro.

\section{Complement C5a mediates the pro-apoptotic effect of DS on cultured hUC-MSCs}

It has been repeatedly confirmed that uncontrolled serum complement activation plays an important role in the pathogenesis of diabetic complications [21-23], while our data (Fig. 1a, b) suggested that fewer circulating MSC-like cells may result in more complications. Hence, we speculated that there may be a correlation between the activation status of the complement system and the abundance of circulating MSClike cells in diabetes. The $\mathrm{CH} 50$ and complement $(\mathrm{C} 3, \mathrm{C} 4$, sC5b-9 and C5a) levels in DS were significantly higher than those in NDS (Table 2), and the abundance of circulating MSC-like cells was negatively correlated with the serum complement activity (ESM Fig. 4). However, a correlation does not imply causality. We then inactivated complement by heating the serum at $56^{\circ} \mathrm{C}$ for $30 \mathrm{~min}$ to determine whether complement played a causal role in mediating the cytotoxicity of DS. The cytotoxic effect of DS on the hUC-MSCs was largely attenuated by the pre-inactivation procedure (Fig. 4a-d). Although the cytotoxicity of DS was much higher than that of NDS, there was no significant difference in the cytotoxicity or pro-apoptotic effect between the heatinactivated DS (HI-DS) and heat-inactivated NDS (HI-NDS) (Fig. 4a-d).

Typically, complement-dependent cytotoxicity occurs upon assembly of the membrane attack complex (MAC) and its insertion into the plasma membrane of target cells [26]. However, by pre-incubating hUC-MSCs with recombinant human CD59 (rhCD59; Sino Biological, Beijing, China) to inhibit the assembly and insertion of the MAC, we identified only a slight but significant decrease in the DS-induced apoptosis of hUC-MSCs (Fig. 4e, f), suggesting that the MAC may not play a major role in this process. In addition, a large amount of anaphylatoxins will be simultaneously generated 
a

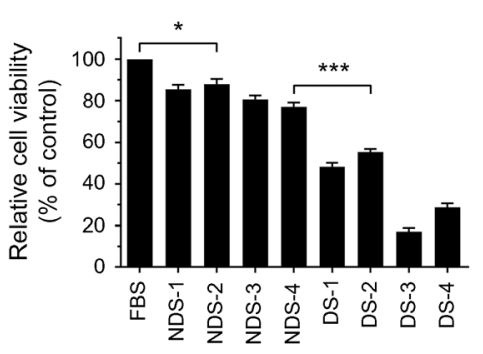

b

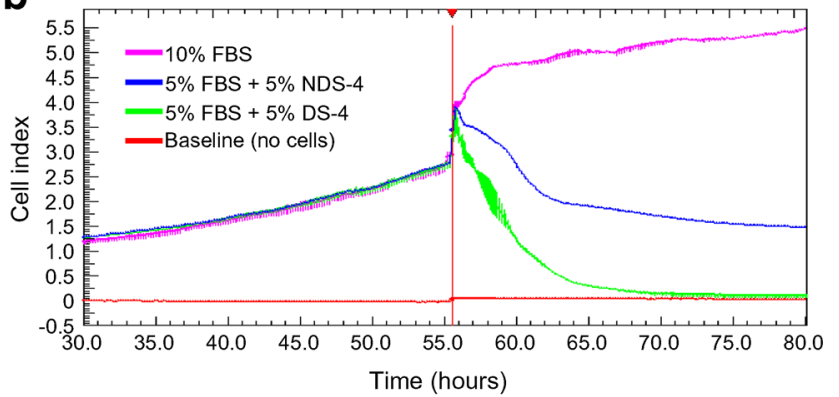

C

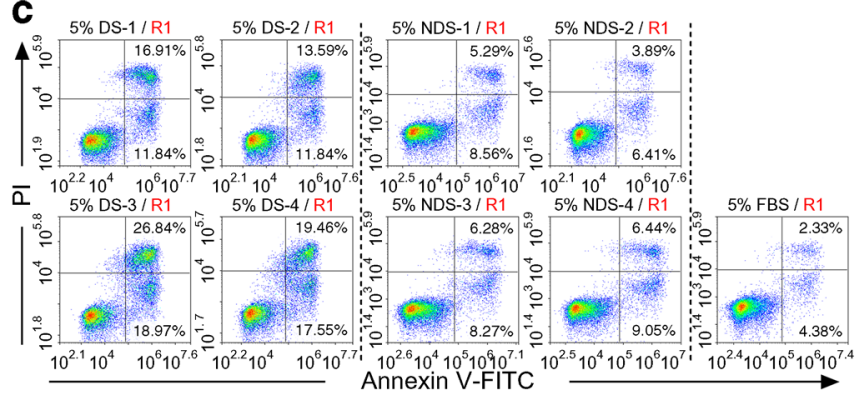

d

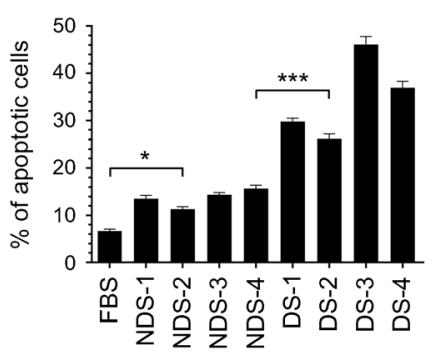

e

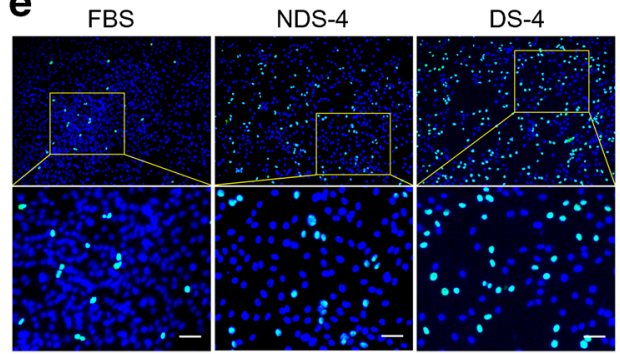

Fig. 2 The effects of NDS or DS on the proliferation and survival of hUCMSCs. (a) The relative cell viability of hUC-MSCs exposed to different sources of sera for $12 \mathrm{~h}$ was determined by MTT assay. DS- and NDS-14: numbered serum samples (randomly selected) from four diabetic and four control participants, respectively. Data are expressed as means \pm SEM $(n=5)$. (b) The adherent areas of hUC-MSCs were continuously monitored by RTCA. Different types of stimulating sera were administered at the indicated time point. Data are expressed as means $\pm \mathrm{SD}(n=2)$. Apoptosis of hUC-MSCs stimulated with the different serum treatments for $12 \mathrm{~h}$ was detected by (c, d) FACS following annexin V/PI double staining $(n=3)$ and $(\mathbf{e}, \mathbf{f})$ the TUNEL assay. (e) Representative photomicrographs of

during complement activation. Of these anaphylatoxins, C5a is the most potent in eliciting biological responses [27, 28], which seems to be important for inducing cell apoptosis [29-32]. As shown in Fig. 4e, f and ESM Fig. 5a, the proapoptotic effect of DS was significantly decreased when it was pre-treated with an anti-C5a antibody (Abcam, Cambridge, $\mathrm{UK} ; 10 \mu \mathrm{g} / \mathrm{ml}$ for $30 \mathrm{~min}$ ) compared with untreated DS. In addition, the addition of recombinant human $\mathrm{C} 5 \mathrm{a}$ (rhC5a; Sino Biological) to HI-DS, which lacks complement activity, partially rescued the pro-apoptotic effect. However, although C5a acts as a critical mediator of the cytotoxicity of DS against hUC-MSCs, C5a alone did not affect the survival and proliferation of hUC-MSCs over the tested concentration range (0-10 $\mu \mathrm{mol} / \mathrm{l})$ (Fig. 4e, f and ESM Fig. 5). f

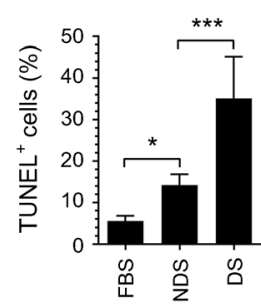

g

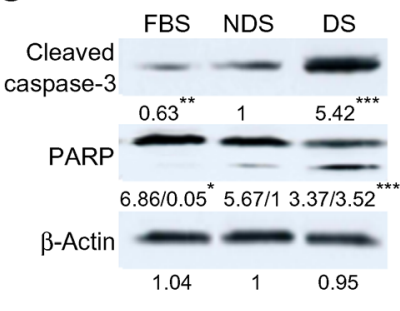

TUNEL staining. Scale bar, $200 \mu \mathrm{m}$. (f) Quantitative evaluation of data from TUNEL assays in which the effects of four independent DS samples and four control NDS samples were evaluated (with three replications). (g) The expression of cleaved caspase-3 and poly-ADP ribose polymerase (PARP) in hUC-MSCs exposed to different types of sera for $12 \mathrm{~h}$ was determined by western blotting. The numbers under each blot represent the relative quantification of the band density, which were calculated as the ratio of the band density of the FBS group or DS group to the band density of the NDS group. Data are from assays in which the effects of four independent DS samples and four control NDS samples were evaluated and are presented as means. $* p<0.05, * * * p<0.001$

\section{The C5a/C5aR pathway plays an essential role in DS-induced apoptosis of MSCs}

C5a exerts its effects through C5aRs, i.e. C5aR1 (CD88) and C5a receptor-like 2 (C5L2). CD88 mediates signalling activity, whereas C5L2 is generally termed a decoy receptor and its function is still enigmatic and controversial [33]. We detected the positive expression of CD88 in both hUC-MSCs and mBMMSCs (Fig. 5a) and further confirmed its location on the surfaces of these cells (Fig. 5b). Moreover, DS induced an increase in CD88 expression in hUC-MSCs (Fig. 5c), indicating that the $\mathrm{C} 5 \mathrm{a} / \mathrm{C} 5 \mathrm{aR}$ pathway had been activated in the DS-exposed hUCMSCs. BM-MSCs derived from $C d 88^{-/}$mice were assessed to determine the role of this pathway in DS-induced apoptosis. We 
a

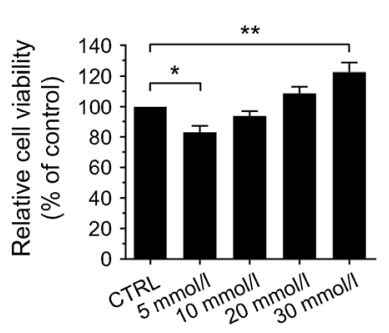

b

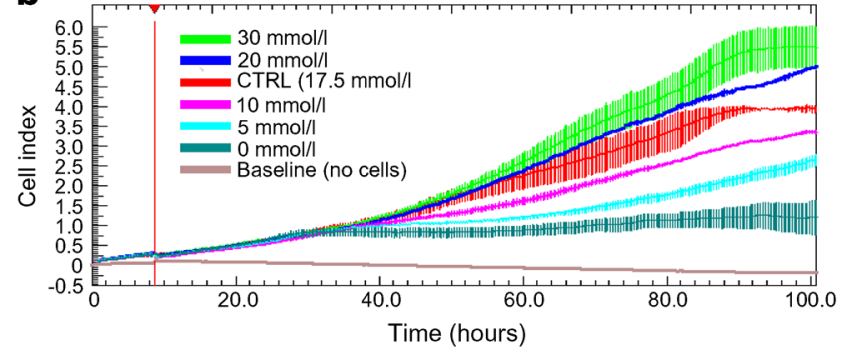

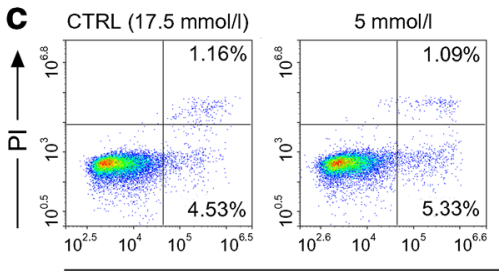
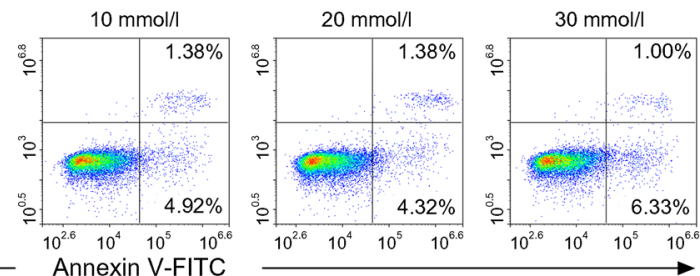

d

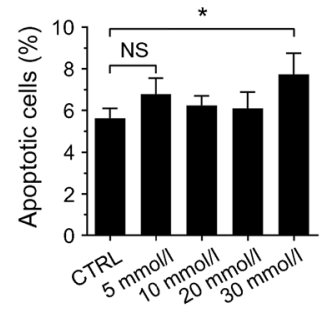

e

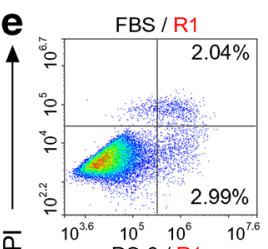

$\overline{\mathbf{L}}$

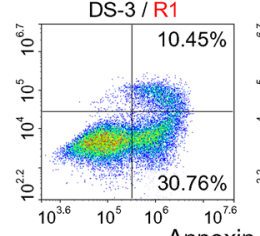

Fig. 3 Effects of different concentrations of D-glucose on the proliferation and survival of hUC-MSCs. (a) The relative cell viability of hUCMSCs exposed to different concentrations of D-glucose for $12 \mathrm{~h}$ was determined by MTT assay $(n=5)$. (b) The effects of different concentrations of D-glucose on the proliferation and viability of hUC-MSCs were continuously monitored by RTCA $(n=2)$. (c, d) The apoptosis of hUCMSCs exposed to different concentrations of D-glucose for $12 \mathrm{~h}$ was detected by FACS following annexin V/PI double staining $(n=3)$. $(\mathbf{e}, \mathbf{f})$

did not observe an obvious increase in the apoptosis of $\mathrm{Cd} 88^{-/}$ mBM-MSCs exposed to DS compared with cells exposed to

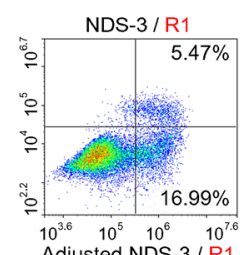

Adjusted NDS-3 / R1

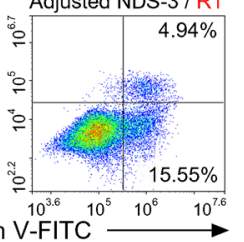

f

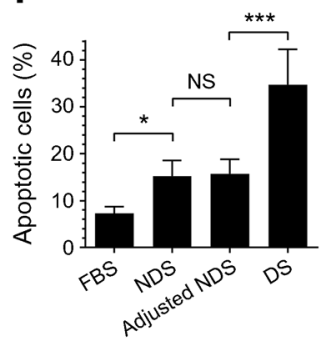

The apoptosis of hUC-MSCs that had been treated with FBS, NDS, adjusted NDS or DS for $12 \mathrm{~h}$ was determined by FACS with three replications: (e) a representative example of results obtained from one set of experiments in which one DS sample and one control NDS sample were used; (f) statistical analysis of the results of FACS analyses in which the effects of four independent DS samples and four control NDS samples were evaluated. Control (CTRL), normal culture condition containing $17.5 \mathrm{mmol} / 1$ D-glucose. $* p<0.05, * * p<0.01, * * * p<0.001$

NDS (Fig. 5d). In addition, following treatment with DS, the expression of pro-apoptotic proteins (Fas-associated protein

Table 2 Serum levels of $\mathrm{CH} 50$, complement components (C3 and $\mathrm{C} 4)$ and activation products (sC5b-9 and $\mathrm{C} 5 \mathrm{a}$ ) in diabetic and control participants

Variable $\quad$ Control group $(n=15) \quad$ Type 2 diabetes group $(n=49)$

$p$ value

\begin{tabular}{lcccc} 
& & 0 complication $(n=14)$ & 1 complication $(n=19)$ & $\geq 2$ complications $(n=16)$ \\
\hline CH50 $(\mathrm{U} / \mathrm{ml})$ & $37.55 \pm 6.86$ & $44.25 \pm 9.79$ & $45.48 \pm 14.09$ & $47.46 \pm 14.17$ \\
$\mathrm{C} 3(\mathrm{~g} / \mathrm{l})$ & $1.01 \pm 0.16$ & $1.10 \pm 0.10$ & $1.14 \pm 0.17$ & $1.17 \pm 0.22^{*}$ \\
$\mathrm{C} 4(\mathrm{~g} / \mathrm{l})$ & $0.31 \pm 0.12$ & $0.38 \pm 0.09$ & $0.40 \pm 0.12$ & $0.41 \pm 0.14$ \\
$\mathrm{sC} 5 \mathrm{~b}-9(\mu \mathrm{g} / \mathrm{l})$ & $83.31 \pm 21.50$ & $108.60 \pm 16.51^{*}$ & $121.90 \pm 26.91^{* * *}$ & $132.40 \pm 29.56 * * *$ \\
$\mathrm{C} 5 \mathrm{a}(\mu \mathrm{g} / \mathrm{l})$ & $6.38 \pm 2.58$ & $14.02 \pm 5.60^{* *}$ & $20.61 \pm 7.62^{* * *}$ & $24.99 \pm 8.90^{* * *}$ \\
\hline
\end{tabular}

Data are presented as means $\pm \mathrm{SD}$

All data were analysed using one-way ANOVA, followed by Dunnett's test for multiple comparisons ${ }^{*} p<0.05, * * p<0.01$ and $* * * p<0.001$ compared with control participants 
a
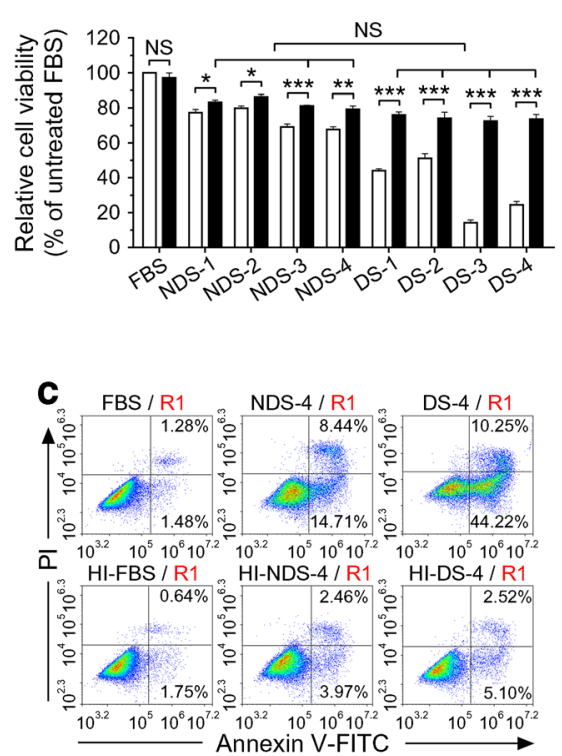

b

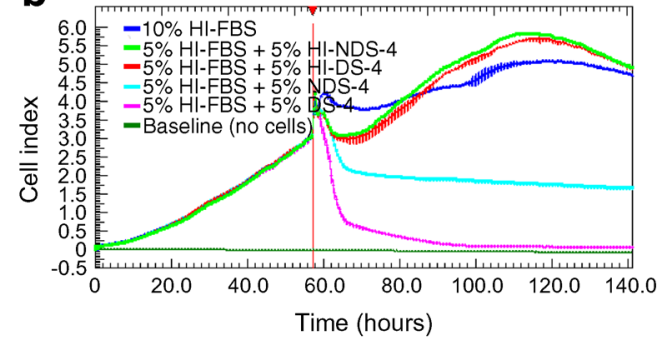

d
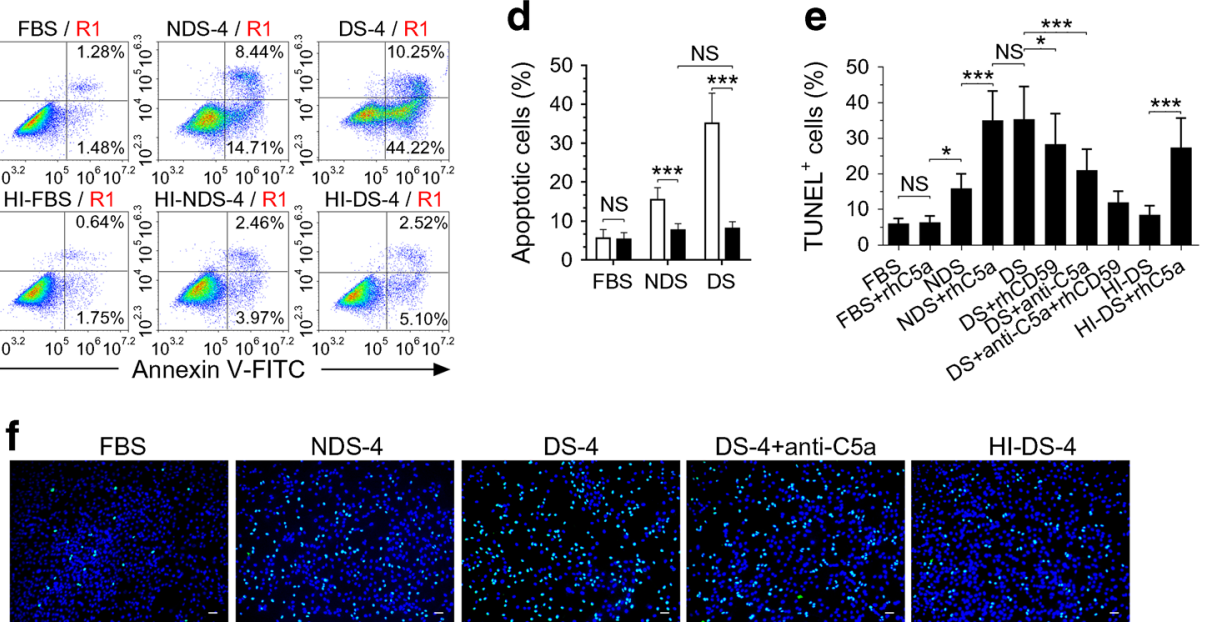

$\mathrm{FBS}+\mathrm{rhC5a}$

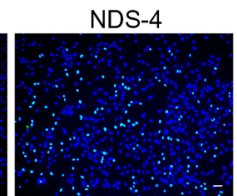

NDS- $4+$ rhC5a
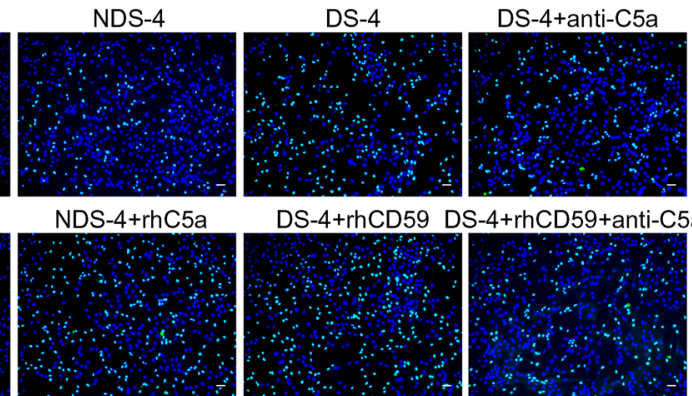

HI-DS-4

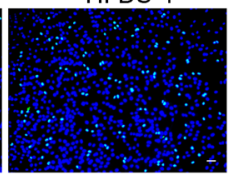

-4+rhCD59+anti-C5a HI-DS-4+rhC5a
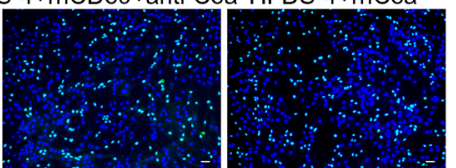

Fig. 4 Complement mediates the cytotoxicity of DS towards hUC-MSCs. (a) The relative cell viability of hUC-MSCs cultured under different serum conditions for $12 \mathrm{~h}$ was determined by MTT assay $(n=5)$. (b) The dynamic effects of untreated or heat-inactivated human serum samples on hUCMSCs were analysed by RTCA $(n=2)$. (c, d) The apoptosis of hUCMSCs exposed to untreated or heat-inactivated sera for $12 \mathrm{~h}$ was determined by FACS following annexin V/PI double staining. (e, f) The effects of blockade of MAC formation or the biological effects of C5a on the

with death domain [FADD], cleaved caspase-3 and caspase-8) and the Bcl-2-associated X protein (BAX)/B cell lymphoma 2 (Bcl-2) ratio were much lower in $\mathrm{Cd} 88^{-/} \mathrm{mBM}-\mathrm{MSCs}$ than in WT mBM-MSCs (Fig. 5e). We further confirmed the regulatory effects of the $\mathrm{C} 5 \mathrm{a} / \mathrm{C} 5 \mathrm{aR}$ pathway on the expression of apoptosis-related proteins in hUC-MSCs by antagonising CD88 (a $30 \mathrm{~min}$ preincubation with $100 \mathrm{nmol} / 1 \mathrm{PMX}-53$ [AcF-(OPdChaWR); GL Biochem, Shanghai, China]) or by the addition of rhC5a (Fig. 5f).

\section{Blockade of the C5a/C5aR pathway inhibits MSC apoptosis in vivo}

As indicated above, MSC apoptosis in diabetes mellitus might be mediated by the $\mathrm{C} 5 \mathrm{a} / \mathrm{C} 5 \mathrm{aR}$ pathway. We transplanted prelabelled BM-MSCs from WT or $C d 88^{-/}$mice into WT or survival of hUC-MSCs exposed to human serum samples were evaluated using the TUNEL assay. Scale bars, $200 \mu \mathrm{m}$. White bars, untreated control; black bars, heat-inactivated samples. (b, c, f) Illustrate the representative results obtained from one set of experiments in which one DS sample and one control NDS sample were used; (d, e) are statistical analyses of the results of FACS analyses or TUNEL assays in which the effects of four independent DS samples and four control NDS samples were evaluated. $* p<0.05, * * p<0.01, * * * p<0.001$

$L e p r^{d b / d b}$ mice and analysed the apoptosis of the transplanted cells to validate this hypothesis in vivo (Fig. 6a). In Lepr ${ }^{d b / d b}$ recipient mice, the rate of $C d 88^{-/-} \mathrm{mBM}-\mathrm{MSC}$ apoptosis was much lower than the rate in the WT mBM-MSCs, and apoptosis of the WT mBM-MSCs was significantly inhibited by daily i.p. injections with $1 \mathrm{mg} / \mathrm{kg}$ PMX-53 starting on the day before transplantation (Fig. 6b). The rate of WT mBM-MSC apoptosis was much higher in $L e p r^{d b / d b}$ recipient mice than in the WT recipient mice, whereas the rates of $C d 88^{-/-} \mathrm{mBM}-$ MSC apoptosis were similar in the WT and $L e p r^{d b / d b}$ recipient mice (Fig. 6c).

\section{Discussion}

In diabetes mellitus, a variety of cells and tissues are affected primarily from damage or dysfunction induced by chronic 

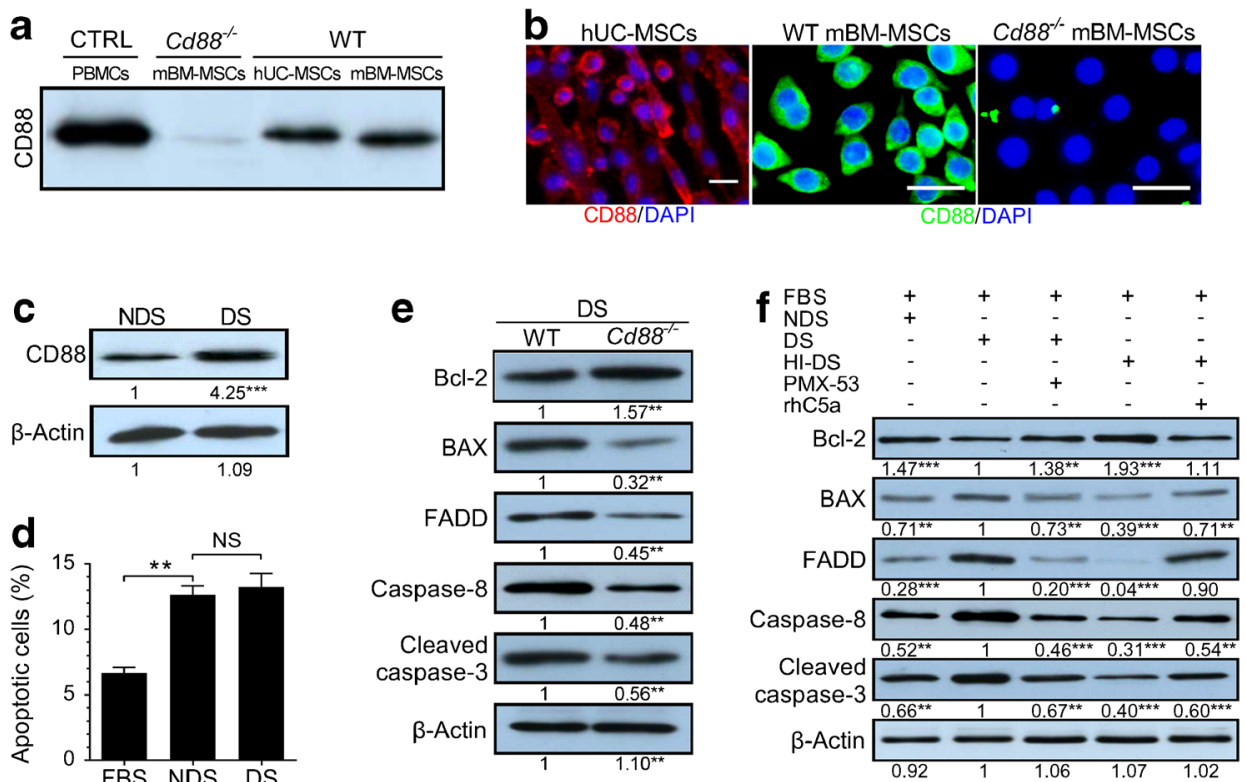

Fig. 5 DS-induced MSC apoptosis depends on the upregulation of FADD and the $\mathrm{BAX} / \mathrm{Bcl}-2$ ratio mediated by the $\mathrm{C} 5 \mathrm{a} / \mathrm{C} 5 \mathrm{aR}$ pathway. (a) The level of CD88 in MSCs was determined by western blotting. (b) Immunocytochemical detection of CD88 on MSCs. Images display both CD88 (red in hUC-MSCs; green in mBM-MSCs) and the nuclear stain DAPI (blue). Scale bars, $100 \mu \mathrm{m}$. (c) The levels of CD88 in hUCMSCs exposed to NDS or DS. (d) The apoptosis of $C d 88^{-/-} \mathrm{mBM}-\mathrm{MSC}$ exposed to the different serum treatments for $12 \mathrm{~h}$ was determined by FACS following annexin V/PI double staining. Data are presented as

hyperglycaemia, and stem cell-dependent regeneration and repair are initiated simultaneously. When regeneration and repair fail to compensate for the damage or dysfunction, complications may arise. MSCs are a population of pluripotent cells with strong capacities for self-renewal, multi-lineage differentiation, paracrine secretion and immunoregulation that are located in virtually all postnatal organs and tissues [34]; thus, these cells have excellent potential for promoting regeneration and repair $[10,35,36]$. In this study, we detected an obvious reduction in the abundance of circulating MSC-like cells in both individuals with type 2 diabetes and diabetic $L e p r^{d b / d b}$ mice, and we proposed that the shortage of functional MSCs might promote the development of diabetic complications and that this deficiency is mainly attributed to an increase in complement-induced apoptotic cell death. This proapoptotic process was at least partially mediated by C5a, which activated the extrinsic and intrinsic apoptotic pathways in MSCs upon binding to C5aR (Fig. 6d). We believe that these findings provide insights into the further management and prevention of diabetic complications.

To improve the detection rate of circulating MSCs, we used a haematopoietic marker (CD34) and a mesenchymal marker (CD105) together to identify circulating MSCs. According to the nomenclature and definition of MSC [37, 38], it would be more meaningful if more detection markers were included. Moreover, we explored the underlying mechanisms using means \pm SEM $(n=4)$. (e) The levels of apoptosis-related proteins in WT or $C d 88^{-/-}$mBM-MSCs exposed to DS for $12 \mathrm{~h}$ detected by western blotting. (f) The levels of apoptosis-related proteins in hUC-MSCs exposed to the indicated conditions for $12 \mathrm{~h}$ measured by western blotting. The numbers under each blot represent the relative quantification of the band density, which were calculated as the ratio of the band density of each group to the band density of the control group. The average grayscale intensities of control bands are denoted as " 1 ", and data are presented as means. $* * p<0.01, * * * p<0.001$

hUC-MSCs and mBM-MSCs instead of peripheral bloodderived MSCs (PB-MSCs), as we failed to culture and expand MSCs from peripheral blood. We believe that the data from PB-MSCs would be more convincing.

Hyperglycaemia is the most prominent characteristic of diabetes, and its effects on the proliferation and survival of MSCs have been repeatedly studied; however, the results of these studies have been contradictory. Two independent teams have reported that high glucose levels induce an increase in MSC apoptosis and a decrease in MSC proliferation [39, 40], whereas two other groups have reported the complete resistance of human MSCs to high glucose toxicity [41, 42]. In addition, Ryu and colleagues have reported that high glucose levels induced MSC proliferation [43]. As shown in our study, high glucose levels promoted hUC-MSC proliferation but did not affect their survival. Nevertheless, the hyperglycaemic condition in diabetes mellitus is chronic and dynamic, whereas the duration of high glucose exposure in our study was no more than 1 week. Therefore, the effects of long-term and fluctuating high glucose levels on the proliferation and survival of MSCs must be studied further.

In the present study, we eliminated the effects of the complement system using a classical heating procedure, but the possibility that some other potential deleterious molecules present in the serum might have been simultaneously inactivated by this heating step cannot be excluded. Moreover, based on the limited 
a

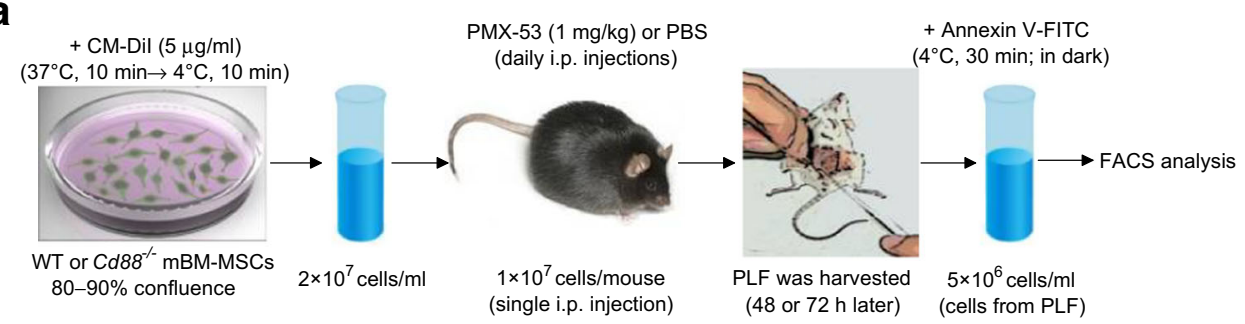

b

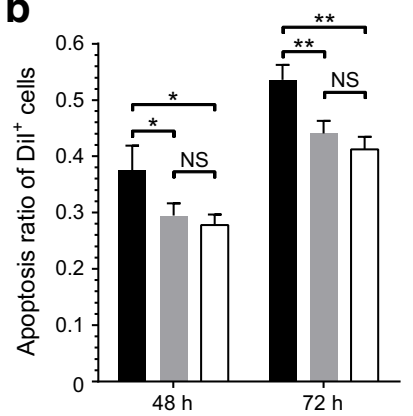

d

C

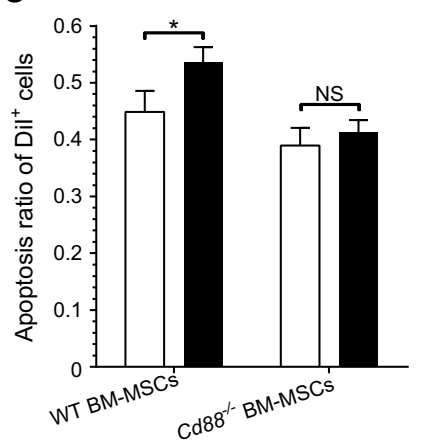

Fig. 6 Survival of the DiI-labelled WT or $C d 88^{-/-}$mBM-MSCs after being transplanted into mice. (a) Flowchart of the animal experiments. (b) Apoptosis of the Dil-labelled WT or $C d 88^{-/-} \mathrm{mBM}-\mathrm{MSCs}$ at $48 \mathrm{~h}$ or $72 \mathrm{~h}$ after being transplanted into Lepr $^{d b / d b}$ recipient mice. White bars, WT mBM-MSCs; grey bars, WT mBM-MSCs + PMX-53; black bars, $C d 88^{-/}$mBM-MSCs. (c) Apoptosis of the DiI-labelled WT or $C d 88^{-/-}$

protective effect of preincubation with rhCD59, we preliminarily conclude that cytolytic MAC formation did not play a central role in the DS-induced death of hUC-MSCs, but further confirmation is required. On one hand, only membrane-bound CD59 has a protective function, but little is known about the membrane insertion efficiency of rhCD59 used in this study. As shown in a previous study, the in vitro activity of rhCD59 against complement-mediated cytolysis is 1000 -fold less than that of rhCD59-P, a genetically modified membrane-targeted recombinant CD59 [44]. On the other hand, further investigations are required to determine whether the pro-apoptotic effect of DS is relevant to sublytic MAC formation because sublytic and nonlytic MAC doses also have pro-apoptotic effects [45-47].

Anaphylatoxin C5a has been proven to be a potent inducer of apoptosis in several cell types [29-32]. Similarly, our data demonstrated the pivotal role of $\mathrm{C} 5 \mathrm{a}$ in mediating the pro-
mBM-MSCs at $72 \mathrm{~h}$ after being transplanted into WT or $L e p r^{d b / d b}$ recipient mice. White bars, WT recipient mice; black bars, $L e p r^{d b / d b}$ recipient mice. (d) Signalling cascades triggered by the activation of C5aR induce MSC apoptosis via both the extrinsic and intrinsic apoptosis pathways. PARP, poly-ADP ribose polymerase; PLF, peritoneal lavage fluid. Data are presented as means $\pm \operatorname{SEM}(n=5) . * p<0.05, * * p<0.01$.

apoptotic effect of DS on MSCs. Thus, we sought to confirm whether C5a alone could induce MSC apoptosis. To our surprise, we did not observe obvious changes in the viability and survival of hUC-MSCs when they were directly challenged with rhC5a. These results implied a synergistic mechanism in the process of DS-induced MSC apoptosis. We conclude that there should be another substance or abnormity in the diabetic environment that promotes the activation of the $\mathrm{C} 5 \mathrm{a} / \mathrm{C} 5 \mathrm{aR}$ pathway and, subsequently, MSC apoptosis.

This study has indicated for the first time that excessive activation of the complement system in the diabetic microenvironment not only inhibits MSC proliferation but also induces MSC apoptosis, a process correlated with the progression of diabetic complications. Notably, both individuals with type 2 diabetes and $L e p r^{d b / d b}$ mice are characterised by obesity, a condition in which increased complement activation has 
also been reported [48]. However, the diabetic and control participants in this study were not matched for BMI, and thus, we could not be sure of the initial cause of complement activation in participants. It is reasonable to speculate that complement-mediated MSC apoptosis may also occur in obesity, and early weight control may be an effective way to maintain an abundance of circulating MSCs.

Acknowledgements We thank the donors and staff from several clinical departments of Daping Hospital (Department of Hypertension and Endocrinology, Health Care Center, and Department of Obstetrics) for providing samples and clinical data, Y. Wang (Department of Medical Genetics, Third Military Medical University, Chongqing, China) for providing the $\mathrm{Lepr}^{\mathrm{db} / \mathrm{+}}$ mice and guidance for mouse identification and breeding, and G. Xu (Institute of Immunology, College of Basic Medical Science, Third Military Medical University, Chongqing, China) for providing the $C d 88^{-/}$mice. We also acknowledge the professional manuscript services of American Journal Experts (Durham, NC, USA).

Data availability The datasets generated during the current study are available from the corresponding author upon reasonable request.

Funding This work was supported by grants from the National Natural Science Foundation of China (No. 81372027 and No. 81571912) and from the Foundational and Cutting-edge Research Plan of Chongqing: Special Projects for Academicians (No. cstc2014jcyjys10002).

Duality of interest The authors declare that there is no duality of interest associated with this manuscript.

Contribution statement $\mathrm{XX}$ and $\mathrm{HH}$ conceived/designed the study, reviewed the manuscript and are responsible for the integrity of this work. $\mathrm{MZ}$ designed the study, performed the experiments, analysed the data and wrote the manuscript. XH, XHW and WQ performed the experiments and analysed the data. WX, WG, TCA and XTH performed the experiments. LQA and ZL contributed to acquisition of clinical data. XPL and NX made substantial contributions to conception and design of the study and edited the manuscript. JY reviewed/edited the manuscript and contributed to the analysis and interpretation of data. All authors revised the manuscript critically for important intellectual content and approved the final version for publication.

\section{References}

1. World Health Organization (2016) Global report on diabetes. World Health Organization, Geneva

2. NCD Risk Factor Collaboration (2016) Worldwide trends in diabetes since 1980: a pooled analysis of 751 population-based studies with $4 \cdot 4$ million participants. Lancet 387:1513-1530

3. Whiting DR, Guariguata L, Weil C, Shaw J (2011) IDF diabetes atlas: global estimates of the prevalence of diabetes for 2011 and 2030. Diabetes Res Clin Pract 94:311-321

4. International Diabetes Federation (2015) IDF diabetes atlas, 7th edn. International Diabetes Federation, Brussels

5. Krug EG (2016) Trends in diabetes: sounding the alarm. Lancet 387:1485-1486

6. Dixon J (2015) The global burden of obesity and diabetes. In: Brethauer A, Schauer R, Schirmer D (eds) Minimally invasive bariatric surgery. Springer, New York, pp 1-6
7. Marcovecchio ML, Lucantoni M, Chiarelli F (2011) Role of chronic and acute hyperglycemia in the development of diabetes complications. Diabetes Technol Ther 13:389-394

8. Brownlee M (2005) The pathobiology of diabetic complications: a unifying mechanism. Diabetes 54:1615-1625

9. Brownlee M (2001) Biochemistry and molecular cell biology of diabetic complications. Nature 414:813-820

10. Volarevic V, Arsenijevic N, Lukic ML, Stojkovic M (2011) Concise review: mesenchymal stem cell treatment of the complications of diabetes mellitus. Stem Cells 29:5-10

11. Wise AF, Ricardo SD (2012) Mesenchymal stem cells in kidney inflammation and repair. Nephrology 17:1-10

12. Trzaska K, Castillo M, Rameshwar P (2008) Adult mesenchymal stem cells in neural regeneration and repair: current advances and future prospects (review). Mol Med Rep 1:307-316

13. Zhang Y, Liang X, Lian Q, Tse H-F (2013) Perspective and challenges of mesenchymal stem cells for cardiovascular regeneration. Expert Rev Cardiovasc Ther 11:505-517

14. Kim Y, Kwon J, Hong M et al (2013) Restoration of angiogenic capacity of diabetes-insulted mesenchymal stem cells by oxytocin. BMC Cell Biol 14:38

15. Rodrigues M, Wong VW, Rennert RC, Davis CR, Longaker MT, Gurtner GC (2015) Progenitor cell dysfunctions underlie some diabetic complications. Am J Pathol 185:2607-2618

16. Yang G, Jia Y, Li C, Cheng Q, Yue W, Pei X (2015) Hyperglycemic stress impairs the stemness capacity of kidney stem cells in rats. PLoS One 10:e139607

17. El-Ftesi S, Chang EI, Longaker MT, Gurtner GC (2009) Aging and diabetes impair the neovascular potential of adipose-derived stromal cells. Plast Reconstr Surg 123:475-485

18. Kim SM, Kim YH, Jun YJ, Yoo G, Rhie JW (2016) The effect of diabetes on the wound healing potential of adipose-tissue derived stem cells. Int Wound J 13:33-41

19. Kondo M, Kamiya H, Himeno T et al (2015) Therapeutic efficacy of bone marrow-derived mononuclear cells in diabetic polyneuropathy is impaired with aging or diabetes. J Diabetes Invest 6:140-149

20. Nowak WN, Borys S, Kusińska K et al (2014) Number of circulating pro-angiogenic cells, growth factor and anti-oxidative gene profiles might be altered in type 2 diabetes with and without diabetic foot syndrome. J Diabetes Invest 5:99-107

21. Qin X, Goldfine A, Krumrei N et al (2004) Glycation inactivation of the complement regulatory protein CD59: a possible role in the pathogenesis of the vascular complications of human diabetes. Diabetes 53:2653-2661

22. Østergaard J, Hansen TK, Thiel S, Flyvbjerg A (2005) Complement activation and diabetic vascular complications. Clin Chim Acta 361:10-19

23. Ghosh P, Sahoo R, Vaidya A, Chorev M, Halperin JA (2015) Role of complement and complement regulatory proteins in the complications of diabetes. Endocr Rev 36:272-288

24. Flyvbjerg A (2010) Diabetic angiopathy, the complement system and the tumor necrosis factor superfamily. Nat Rev Endocrinol 6: 94-101

25. Lachmann P-J (2010) Preparing serum for functional complement assays. J Immunol Methods 352:195-197

26. Muller-Eberhard HJ (1986) The membrane attack complex of complement. Annu Rev Immunol 4:503-528

27. Ember J, Jagels M, Hugli T (1998) Characterization of complement anaphylatoxins and their biological responses. In: Volanakis JE, Frank MM (eds) Human complement system in health and disease. CRC Press, New York, pp 241-284

28. Köhl J (2001) Anaphylatoxins and infectious and non-infectious inflammatory diseases. Mol Immunol 38:175-187

29. Guo R-F, Huber-Lang M, Wang X et al (2000) Protective effects of anti-C5a in sepsis-induced thymocyte apoptosis. J Clin Invest 106: $1271-1280$ 
30. Flierl MA, Rittirsch D, Chen AJ et al (2008) The complement anaphylatoxin $\mathrm{C} 5 \mathrm{a}$ induces apoptosis in adrenomedullary cells during experimental sepsis. PLoS One 3:e2560

31. Pavlovski D, Thundyil J, Monk PN, Wetsel RA, Taylor SM, Woodruff TM (2012) Generation of complement component C5a by ischemic neurons promotes neuronal apoptosis. FASEB J 26: 3680-3690

32. Hu R, Chen ZF, Yan J et al (2014) Complement C5a exacerbates acute lung injury induced through autophagy-mediated alveolar macrophage apoptosis. Cell Death Dis 5:e1330

33. Li R, Coulthard LG, Wu MC, Taylor SM, Woodruff TM (2013) C5L2: a controversial receptor of complement anaphylatoxin, C5a. FASEB J 27:855-864

34. Porada C, Zanjani E, Almeida-Porada G (2006) Adult mesenchymal stem cells: a pluripotent population with multiple applications. CSCR 1:365-369

35. DiMarino AM, Caplan AI, Bonfield TL (2013) Mesenchymal stem cells in tissue repair. Front Immunol 4:201

36. Mundra V, Gerling IC, Mahato RI (2013) Mesenchymal stem cellbased therapy. Mol Pharm 10:77-89

37. Dominici M, Le Blanc K, Mueller I et al (2006) Minimal criteria for defining multipotent mesenchymal stromal cells. The International Society for Cellular Therapy position statement. Cytotherapy 8: 315-317

38. West MD, Nasonkin I, Larocca D et al (2016) Adult versus pluripotent stem cell-derived mesenchymal stem cells: the need for more precise nomenclature. Curr Stem Cell Rep 2:299-303

39. Stolzing A, Coleman N, Scutt A (2006) Glucose-induced replicative senescence in mesenchymal stem cells. Rejuvenation Res 9: 31-35

40. Li W-T, Hu W-K, Ho F-M (2013) High glucose induced bone loss via attenuating the proliferation and osteoblastogenesis and enhancing adipogenesis of bone marrow mesenchymal stem cells. Biomed Eng Appl Basis Commun 25:1340010

41. Li Y-M, Schilling T, Benisch P et al (2007) Effects of high glucose on mesenchymal stem cell proliferation and differentiation. Biochem Biophys Res Commun 363:209-215

42. Weil BR, Abarbanell AM, Herrmann JL, Wang Y, Meldrum DR (2009) High glucose concentration in cell culture medium does not acutely affect human mesenchymal stem cell growth factor production or proliferation. Am J Phys Regul Integr Comp Phys 296: R1735-R1743

43. Ryu JM, Lee MY, Yun SP, Han HJ (2010) High glucose regulates cyclin D1/E of human mesenchymal stem cells through TGF$\beta 1$ expression via $\mathrm{Ca} 2+/ \mathrm{PKC} / \mathrm{MAPKs}$ and $\mathrm{PI} 3 \mathrm{~K} / \mathrm{Akt} / \mathrm{mTOR}$ signal pathways. J Cell Physiol 224:59-70

44. Hill A, Ridley S, Esser D et al (2006) Protection of erythrocytes from human complement-mediated lysis by membrane-targeted recombinant soluble CD59: a new approach to PNH therapy. Blood 107:2131-2137

45. Hughes J, Nangaku M, Alpers C, Shankland S, Couser W, Johnson R (2000) C5b-9 membrane attack complex mediates endothelial cell apoptosis in experimental glomerulonephritis. Am J Physiol Ren Physiol 278:F747-F757

46. Nauta AJ, Daha MR, Tijsma O, van de Water B, Tedeco F, Roos A (2002) The membrane attack complex of complement induces caspase activation and apoptosis. Eur J Immunol 32:783

47. Pippin JW, Durvasula R, Petermann A, Hiromura K, Couser WG, Shankland SJ (2003) DNA damage is a novel response to sublytic complement C5b-9-induced injury in podocytes. J Clin Invest 111: 877-885

48. Vlaicu SI, Tatomir A, Boodhoo D et al (2016) The role of complement system in adipose tissue-related inflammation. Immunol Res 64:653-664 